\title{
Reseña de ICTS and Innovation for Didactics of Social Sciences
}

Review of ICTs and Innovation for Didactics of Social Sciences

Concepción Fuentes-Moreno*

Autor: Emilio José Delgado-Algarra

Título: ICTs and Innovation for Didactics of Social Sciences

Edición: IGI Global

Lugar de publicación: Hershey (PA, Estados Unidos)

Año: 2020

Idioma: Inglés

ISBN: 9781799828822 (hardcover) / 9781799828839 (paperback) / 9781799828846 (ebook)

Páginas: 295

Esta obra tiene como finalidad promover un uso motivador, funcional y racional de las tecnologías para lograr la consecución de competencias más profundas y significativas en la enseñanza y el aprendizaje de las Ciencias Sociales.

El autor es profesor de Didáctica de la Ciencias Sociales en la Universidad de Huelva y cuenta con una amplia y muy interesante trayectoria docente e investigadora que se refleja en este libro claramente, ya que cada capítulo resulta útil en ambos aspectos. La presentación de cada tema permite contextualizar y comprender en qué consiste cada recurso e incorpora una completa y actualizada revisión bibliográfica a nivel internacional que puede servir de base para iniciarse en la investigación en este ámbito. A pesar de las reflexiones teóricas de primer nivel, no pierde de vista su utilidad para el profesorado en activo y en formación, destinatarios últimos de la obra de Emilio Delgado, por lo que puede ser utilizado como manual universitario en asignaturas de Didáctica de las Ciencias Sociales tanto en grados como en Másteres de formación de profesorado y de investigación educativa.

Se estructura en los siguientes capítulos:

\footnotetext{
*Universitat de Barcelona•ORCID 0000-0002-5467-8090• conchafuentes@ub.edu

Fuentes-Moreno, C. (2020). Reseña de ICTs and innovation for didactics of social sciences. CLIO. History and History teaching, 46, 336-338. https://doi.org/10.26754/ojs clio/clio.2020465336. Recibido: 28/11/2020. Aceptado: 16/12/2020.
} 
1. La enseñanza de las ciencias sociales, la geografía y la historia basada en la investigación: innovación y desarrollo profesional

2. Gamificación y aprendizaje basado en juegos: motivar la educación en ciencias sociales

3. Realidad Aumentada en la Educación en Ciencias Sociales: Integrando Capas Virtuales con la Realidad Física

4. Realidad virtual, recreaciones $3 \mathrm{D}$ e impresión $3 \mathrm{D}$ en la educación en ciencias sociales: creación e interacción con mundos virtuales

5. Sistemas de Gestión del Aprendizaje y Recursos Educativos Abiertos para la Enseñanza de las Ciencias Sociales: Monitoreo de Estudiantes e Interacción Virtual

6. Cursos online abiertos masivos como un tipo específico de e-Leaning: del envío de información al intercambio de conocimientos

7. B-Learning y el aula invertida en la educación en ciencias sociales: la escuela como espacio colaborativo de aprendizaje

8. M-Learning como motor del compromiso social, la colaboración responsable y la interacción de los estudiantes: recursos para la educación en ciencias sociales

9. U-Learning y aulas virtuales en la educación en ciencias sociales: entornos de aprendizaje virtual y participación

10. El futuro de las TIC / LKT en la enseñanza y el aprendizaje de las ciencias sociales, la geografía y la historia: aprendizaje adaptativo e inteligencia artificial

El libro contribuye, sin duda alguna, a propiciar un cambio de dinámicas y procesos en cuanto al uso de las TIC para el aprendizaje de las Ciencias Sociales, pues incluye recursos y estrategias orientadas a Educación Primaria y Secundaria, abordando la utilización de realidad virtual, realidad aumentada, videojuegos, impresión 3D, mlearning, b-learning, aula virtual, etc. Constituye al mismo tiempo una guía inicial para la investigación, un manual universitario y una compilación de buenas prácticas, recursos y propuestas para el aula, que resultará de especial interés a quienes quieran 
familiarizarse con tendencias emergentes tecnológicamente en su aplicación a la enseñanza de las Ciencias Sociales como las Tecnologías de Aprendizaje Adaptativo, Inteligencia Artificial o Robótica y en general a toda aquella persona que quiera conocer los recursos tecnológicos y las estrategias innovadoras para la didáctica de las ciencias sociales. 